\title{
Assignment of CPS1, OTC, CRYD2, ARG2 and $A S S$ genes to the chicken RH map
}

\author{
Takeshi SHIMOGIRI $^{\mathrm{a}}$, Natalia BosAK ${ }^{\mathrm{b}}$, Mireille Morisson ${ }^{\mathrm{c}}$, \\ Shin OKамото ${ }^{a}$, Kotaro KawABE ${ }^{\mathrm{d}}$, Yoshizane MAEDA ${ }^{\mathrm{a}}$, \\ Alain VIGNAL ${ }^{\mathrm{c}}$, Hiroshi YASUE ${ }^{\mathrm{b} *}$ \\ ${ }^{a}$ Faculty of Agriculture, Kagoshima University, Kagoshima, Japan \\ ${ }^{\mathrm{b}}$ Genome Research Department, National Institute of Agrobiological Sciences, \\ Tsukuba, Japan \\ ${ }^{\mathrm{c}}$ Laboratoire de génétique cellulaire, Institut national de la recherche agronomique, \\ Castanet-Tolosan, France \\ ${ }^{\mathrm{d}}$ Gene Research Center, Kagoshima University, Kagoshima, Japan
}

(Received 30 October 2003; accepted 27 April 2004)

\begin{abstract}
An attempt was made to assign five genes, CPS1, OTC, ASS, CRYD2, and ARG2, to chicken chromosomes (GGA) by radiation-hybrid mapping. OTC was assigned to GGA1; $A R G 2$ to GGA5; CPS1 to GGA7; and CRYD2 to GGA19. ASS was not, however, assigned to a specific chromosomal position.
\end{abstract}

ornithine-urea cycle / chicken / radiation hybrid mapping / chromosomal assignment

\section{INTRODUCTION}

The ornithine-urea cycle is an enzyme system that functions in the biosynthesis of urea. This cycle consists of five enzymes, carbamyl phosphate synthetase I (CPS1), ornithine transcarbamylase (OTC), argininosuccinate lyase (ASL), argininosuccinate synthetase (ASS), and arginase (ARG). The ornithine-urea cycle has been shown to be conserved in many organisms from bacteria including $E$. coli to mammalian species, and is involved in key physiological functions [2]. All animal species on land except for birds and reptiles so far examined are found to use the cycle for nitrogen excretion.

Birds and reptiles are uricotelic in terms of nitrogen excretion, so that they apparently do not require the ornithine-urea cycle for nitrogen excretion. However, the activities of OTC, ASL, ASS and ARG have been reported in the

\footnotetext{
*Corresponding author: hyasue@affrc.go.jp
} 
chicken kidney [15]. In the chicken, ASL activity was found in the product of the $\delta 2$-crystallin (CRYD2) gene, showing that CRYD2 in the chicken is the ortholog of ASL [9, 13]. Concerning CPS1, Tamir and Ratner [15] reported no enzyme activity in the chicken liver, kidney, spleen, and pancreas; this is consistent with the fact that the ornithine-urea cycle is not involved in nitrogen excretion.

In the present study, in order to characterize the "ornithine-urea cycle" genes in the chicken, we first attempted to obtain a sequence expressed from CPS1 as well as a part of its chicken genomic sequence, and to assign the genes to chicken chromosome (GGA) by radiation-hybrid $(\mathrm{RH})$ mapping.

\section{MATERIALS AND METHODS}

\subsection{Primers}

Primer pairs for $O T C, C R Y D 2$, and $A S S$ were designed based on the chicken EST shown in Table I. For ARG, two isoforms were reported: one was derived from the arginase I gene $(A R G 1)$ and the other from the arginase II gene (ARG2) [4]. ARG1 is expressed in the liver, whereas $A R G 2$ is expressed in extrahepatic tissues including the kidney [6]. Since the "ornithine-urea cycle" enzymes were found in the chicken kidney [15], the primer pair was designed for $A R G 2$.

A cDNA of CPS1 was isolated from the mRNA of a chick embryo at day

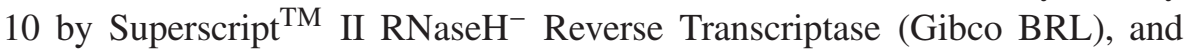
then by semi-nested PCR using primer pairs designed with the consensus sequence of the human and frog genes (Genbank accession number AF154830 and U05193), followed by 5'/3' rapid amplification of cDNA ends (RACE) (the Marathon ${ }^{\mathrm{TM}}$ cDNA amplification kit; Clontech, CA, USA). The cDNA thus obtained was sequenced to confirm that the cDNA was derived from the chicken ortholog by comparison with those of the human and frog, and then the primer-pair for CPS1 was redesigned inside of the sequence. Since the exon-intron structure was not reported for the sequences of CPS1, ASS, and $A R G 2$, primer pairs for the respective genes were designed, assuming that the exon-intron structures of those genes were the same as those of humans.

The DNA fragments amplified from the chicken genomic DNA by the respective primer pairs were directly sequenced using an ABI PRISM 3100 Genetic Analyzer (Applied Biosystems, CA, USA), and compared with the sequences used for the primer design to confirm that the chicken fragments had the expected sequences. 
Table I. The primer-pairs for CPS1, OTC, CRDYD2, ARG2, and ASS.

\begin{tabular}{|c|c|c|c|c|c|c|}
\hline Gene symbol & Reference $^{\mathrm{a}}$ & Primer $\left(5^{\prime}-3^{\prime}\right)$ & $\mathrm{Tm}^{\mathrm{b}}$ & $\begin{array}{c}\text { Cycle } \\
\text { number }\end{array}$ & Product $^{\mathrm{a}}$ & $\mathrm{HSA}^{\mathrm{c}}$ \\
\hline CPS1 & AB159266 & $\begin{array}{l}\text { TCCGAGCCAGACACTCACTA } \\
\text { CTTGGATGCCAAAGCTGAAC }\end{array}$ & 61 & 35 & AY435514 & 2q35 \\
\hline OTC & AF065629 & $\begin{array}{l}\text { GTCATGGTTTCCCTGCTGAC } \\
\text { ATCATTCGTTGCCTTGATCC }\end{array}$ & 61 & 35 & AB159220 & Xp21.1 \\
\hline$C R Y D 2^{\mathrm{d}}$ & M10806 & $\begin{array}{l}\text { GAGATAAACTCTGGGGGGGAA } \\
\text { GGATGCCAGCCTTCTCCAGT }\end{array}$ & 59 & 40 & AB159221 & $\begin{array}{l}\text { 7cen-q11.2 } \\
\text { (ASL) }\end{array}$ \\
\hline$A R G 2$ & EST & GCCAACTGTACGACTTTGGAG & 63 & 35 & AB159222 & $14 \mathrm{q} 24.1-\mathrm{q} 24.3$ \\
\hline$A S S$ & $\begin{array}{l}356527.4^{\mathrm{e}} \\
\text { EST }\end{array}$ & $\begin{array}{l}\text { AGCTGTGTCCAGCAGCTACC } \\
\text { CAGGATGTCTGCAGGGAGTT }\end{array}$ & 63 & 35 & AB159223 & $9 \mathrm{q} 34.1$ \\
\hline
\end{tabular}

${ }^{\mathrm{a}}$ Genbank accession number; ${ }^{\mathrm{b}}$ annealing temperature; ${ }^{\mathrm{c}}$ human chromosomal location; ${ }^{\mathrm{d}}$ CRYD2 is the chicken ortholog of human ASL; ${ }^{\mathrm{e}}$ the chicken EST were obtained from BBSRC ChickEST Database [1]. 


\subsection{Radiation Hybrid (RH) mapping}

A whole genome chicken/hamster RH panel (ChickRH6), which was constructed by Morisson et al. [11], was used for RH mapping of the five genes. The ChickRH6 is composed of 90 clones, whose average retention frequency is $22 \%$ [11]. Although comprehensive RH maps of the chicken genome have not yet been constructed using ChickRH6, 1342 markers have been genotyped. The large chromosomes (GGA1 to GGA7 and GGAZ) are covered with an average 106 markers and 7 microchromosomes with an average 30 markers. Framework maps were built for GGA2, GGA5, GGA7, GGA14 and GGA15 [5] (unpublished data).

For the RH mapping, PCR was performed, essentially following the procedure described by Morisson et al. [11]. The annealing temperature for the PCR and the PCR cycles for each primer-pair are described in Table I. The results were used to assign the five genes via a mapping tool which is a clone of the porcine IMpRH server [10]. Distances and log of odds (lod) scores were calculated according to Lange et al. [7] relative to all markers already genotyped on the panel. We assumed random breakage along the chromosomes and equiprobable retention of fragments.

\section{RESULTS AND DISCUSSION}

\subsection{Sequence identity}

The sequences of the chicken DNA fragments amplified in the PCR using the respective primer-pairs except for CPS1 were compared with the sequences used for the primer design, confirming that the primer-pairs amplified the sequence of the corresponding genes. Genbank accession numbers of these sequences are shown in Table I. Concerning CPS1, since the sequence of chicken CPS1 has not been reported, a 5004-bp cDNA sequence (Accession No. AB159266) of chicken CPS1 was isolated using the semi-nested PCR and 5'/3' RACE methods, and compared with those of human and frog CPS1, which revealed that the similarities between the chicken and human, and between the chicken and frog were $81 \%$ (E-value: $\mathrm{e}^{-119}$ in the Blastn analysis) and $73 \%$, respectively. These similarities led us to conclude that the cDNA was derived from the chicken ortholog of human/frog CPS1. Then, based on the cDNA sequence, primer-pairs for RH mapping of CPS1 were designed and the sequence of the fragment (AY435514) amplified from chicken genomic DNA using the primer-pair was confirmed to be identical to that of cDNA. 
Table II. Results of RH mapping using the ChickRH6 panel DNA.

\begin{tabular}{|c|c|c|c|c|c|c|c|}
\hline \multirow{2}{*}{$\begin{array}{c}\text { Gene } \\
\text { symbol }\end{array}$} & \multirow{2}{*}{$\mathrm{R} \mathrm{F}^{\mathrm{a}}$} & \multirow{2}{*}{$\mathrm{GGA}^{\mathrm{b}}$} & \multicolumn{5}{|c|}{ Nearest markers } \\
\hline & & & Locus $^{c}$ & $\mathrm{R} \mathrm{F}^{\mathrm{a}}$ & $\mathrm{GGA}^{\mathrm{b}}$ & $\mathrm{LOD}^{\mathrm{d}}$ & $\mathrm{HSA}^{\mathrm{b}}$ \\
\hline \multirow[t]{2}{*}{ CPS1 } & 0.34 & 07 & LANCLI & 0.33 & 07 & 19.22 & $2 q 34$ \\
\hline & & & NCKAPI & 0.31 & 07 & 16.49 & $2 q 32$ \\
\hline \multirow[t]{2}{*}{ OTC } & 0.13 & 01 & $U 2 A F 1$ & 0.07 & 01 & 5.96 & $21 \mathrm{q} 22.3$ \\
\hline & & & $D Y R K 1 A$ & 0.11 & 01 & 4.51 & $21 \mathrm{q} 22.13$ \\
\hline \multirow[t]{2}{*}{ CRYD2 } & 0.20 & 19 & $C R K$ & 0.21 & 19 & 14.16 & $17 \mathrm{p} 13.3$ \\
\hline & & & HSA277841 & 0.20 & 19 & 13.77 & $17 \mathrm{p} 13.3$ \\
\hline \multirow[t]{2}{*}{$A R G 2$} & 0.19 & 05 & MPP5 & 0.19 & 05 & 17.94 & $14 q 24.1$ \\
\hline & & & MCW0078 & 0.19 & 05 & 14.13 & Not assigned \\
\hline$A S S$ & 0.17 & Not assigned & & & & & \\
\hline
\end{tabular}

${ }^{a}$ RF represents retention frequency of the marker in the panel; ${ }^{b}$ GGA and HSA, a chicken chromosome and human chromosome, respectively; ${ }^{\mathrm{c}}$ upper and lower rows in each gene indicate the nearest marker and the 2 nd nearest marker, respectively; ${ }^{\mathrm{d}}$ LOD shows a two-point lod score.

\subsection{RH mapping}

Following the procedure described above, we attempted to assign the five genes, CPS1, OTC, CRYD2, ARG2, and ASS to GGA, using the respective primer-pairs and the ChickRH6.

CPS1 was calculated to link to $L A N C L 1$ with the highest lod score of 19.22 , and to NCKAP1 with the second highest lod score of 16.49; LANCL1 and NCKAPI are located on GGA7 (Tab. II). These results, together with the fact that the retention frequency of $C P S 1$ was close to those for $L A N C L 1$ and NCKAP1, indicate that CPS1 resides on GGA7. This finding is consistent with the linkage analysis of CPS1 using the Kobe University resource family [8] and using the East Lancing reference population [3] performed in our laboratory (unpublished data). The localization of CPS1, LANCL1, and NCKAP1 on GGA7 suggests that the conservation of synteny exists in the chromosomal segment present on human chromosome (HSA) 2.

OTC was calculated to link to $U 2 A F 1$ with the highest lod score of 5.96 and to DYRK1A with the second highest lod score of 4.51. Both genes are located on GGA1, indicating that $O T C$ resides on GGA1 (Tab. II). The similarity of the retention frequencies of $O T C$ and $U 2 A F 1$ supports this indication. This result is consistent with the location of OTC in our previous linkage analysis [14]. OTC is localized in HSAXp21.1, whereas U2AF1 and DYRK1A in the proximity of $O T C$ in GGA1 are localized in HSA21q22. These findings indicate that GGA1 corresponds at least to HSAX and HSA21. 
$C R Y D 2$ was calculated to link to $C R K$ with the highest lod score of 14.16 and to HSA277841 with the second highest lod score of 13.77. Both are located in GGA19, indicating that CRYD2 resides on GGA19 (Tab. II). This observation was supported by the similarity of the retention frequencies for $C R Y D 2$, $C R K$, and HSA277841. ASL, the ortholog of CRYD2, is located on HSA7cenq11.2, whereas $C R K$ and HSA277841 in the proximity of CRYD2 in GGA19 are localized in HSA17p13.3; this indicates that GGA19 corresponds at least to HSA7 and HSA17.

$A R G 2$ was calculated to link to MPP5 with the highest lod score of 17.94 and to $M C W 0078$ with the second highest lod score of 14.13. Both are located in GGA5, indicating that $A R G 2$ resides on GGA5 (Tab. II). This observation was supported by the similarity of the retention frequencies for ARG2, MPP5, and $M C W 0078$. It also shows that GGA5 corresponds at least to HSA14.

$A S S$, which is localized in HSA9q34.1, was not linked to any framework markers with lod scores greater than 3 (the threshold of significance) (Tab. II). Therefore ASS could not be assigned to a specific chromosomal region. Nanda et al. [12] reported that HSA9q32-qter showed a conserved synteny with the chicken linkage group E41W17 localizing in GGA17 (http://www.thearkdb.org/). Currently, no markers representing GGA17 have been obtained for the chicken RH map. These facts together with the present findings indicate that ASS could be the first marker for GGA17 in the chicken RH map.

\subsection{Conclusion}

In this study, we first revealed the existence of the CPS1 sequence orthologous to the human/frog CPS1 sequence and successfully assigned all the genes of "ornithine-urea cycle" enzymes except for ASS to GGA using ChickRH6. Since all the "ornithine-urea cycle" genes were found in chicken genomes, a future study should examine whether these genes function together in a physiological process such as the ornithine-urea cycle in mammalian species or whether the genes function independently in various physiological processes.

\section{REFERENCES}

[1] Boardman P.E., Sanz-Ezquerro J., Overton I.M., Burt D.W., Bosch E., Fong W.T., Tickle C., Brown W.R.A., Wilson S.A., Hubbard S.J., A comprehensive collection of chicken cDNAs, Curr. Biol. 12 (2002) 1965-1969. 
[2] Campbell J.W., Excretory nitrogen metabolism, in: Prosser C.L. (Ed.), Environmental and Metabolic Animal Physiology (Comparative Animal Physiology, 4th edn.), Wiley-Liss Inc., New York, 1991, pp. 277-324.

[3] Crittenden L.B., Provencher L., Levin I., Abplanalp H., Briles R.W., Briles W.E., Dodgson J.B., Characterization of a red jungle fowl by White Leghorn backcross reference population for molecular mapping of the chicken genome, Poult. Sci. 72 (1993) 334-348.

[4] Helzfeld A., Raper S.M., The heterogeneity of arginases in rat tissues, Biochem. J. 153 (1976) 469-478.

[5] Jennen D.G.J., Crooijmans R.P.M.A., Morisson M., Grootemaat A.E., Van Der Poel J.J., Vignal A., Groenen M.A.M., A radiation hybrid map of chicken chromosome 15, Anim. Genet. 35 (2004) 63-65.

[6] Kaysen G.A., Strecker H.J., Purification and properties of arginase of rat kidney, Biochem. J. 133 (1973) 779-788.

[7] Lange K., Boehnke M., Cox D.R., Lunetta K.L., Statistical methods for polyploid radiation hybrid mapping, Genome Res. 2 (1995) 136-150.

[8] Lee E.J., Yoshizawa K., Mannen H., Kikuchi H., Kikuchi T., Mizutani M., Tsuji S., Localization of the muscular dystrophy AM locus using a chicken linkage map constructed with the Kobe University resource family, Anim. Genet. 33 (2002) 42-48.

[9] Matsubasa T., Takiguchi M., Amaya Y., Matsuda I., Mori M., Structure of the rat argininosuccinate lyase gene: close similarity to chicken $\delta$-crystallin genes, Proc. Natl. Acad. Sci. USA 86 (1989) 592-596.

[10] Milan D., Hawken R., Cabau C., Leroux S., Genet C., Lahbib Y., Tosser G., Robic A., Hatey F., Alexander L., Beattie C., Schook L., Yerle M., Gellin J., IMpRH server: an RH mapping server available on the Web, Bioinformatics 6 (2000) 558-559.

[11] Morisson M., Lemiere A., Bosc S., Galan M., Plisson-Petit F., Pinton P., Delcros C., Feve K., Pitel F., Fillon V., Yerle M., Vignal A., ChickRH6: a chicken wholegenome radiation hybrid panel, Genet. Sel. Evol. 34 (2002) 521-533.

[12] Nanda I., Shan Z., Schartl M., Burt D.W., Koehler M., Nothwang H., Grutzner F., Paton I.R., Windsor D., Dunn I., Engel W., Staeheli P., Mizuno S., Haaf T., Schmid M., 300 million years of conserved synteny between chicken $\mathrm{Z}$ and human chromosome 9, Nat. Genet. 21 (1999) 258-259.

[13] Piatigorsky J., O’Brien W.E., Norman B.L., Kalumuck K., Wistow G.J., Borras T., Nickerson J.M., Wawrousek E.F., Gene sharing by $\delta$-crystallin and argininosuccinate lyase, Proc. Natl. Acad. Sci. USA 85 (1988) 3479-3483.

[14] Shimogiri T., Kono M., Mannen H., Mizutani M., Tsuji S., Chicken ornithine transcarbamylase gene, structure, regulation, and chromosomal assignment: repetitive sequence motif in intron 3 regulates this enzyme activity, J. Biochem. 124 (1998) 962-971.

[15] Tamir H., Ratner S., Enzymes of arginine metabolism in chicks, Arch. Biochem. Biophys. 102 (1963) 249-258. 